\title{
Telemedicine: an enhanced emergency care program for older adults
}

This article was published in the following Dove Press journal:

Smart Homecare Technology and TeleHealth

2 July 2014

Number of times this article has been viewed

\author{
Paul Y Takahashi' \\ Anupam Chandra' \\ Frederick North' \\ Jennifer L Pecina ${ }^{2}$ \\ Benjavan Upatising ${ }^{3}$ \\ Gregory J Hanson' \\ 'Mayo Clinic Division of Primary \\ Care Internal Medicine, ${ }^{2}$ Mayo \\ Clinic Department of Family \\ Medicine, Rochester, MN, USA; \\ ${ }^{3}$ Regenstrief Center for Healthcare \\ Engineering, Purdue University, \\ West Lafayette, IN, USA
}

\begin{abstract}
Recent changes and consolidations in health care systems have resulted in an increase in new health care delivery models. Telemedicine holds great promise as one of these models. There is a great potential for new patient evaluation and treatment models in emergency care (EC), especially when patients are miles away from a medical team. Evaluations can be performed in a patient's home, a nursing care facility, and in hospitals that focus on advanced subspecialty care. Due to rapid developments in this area, current care models are constantly being evaluated and modified. This review article outlines current telemedicine models for EC and summarizes their potential benefits to patients and the health care system. The review examines the role that the telephone, a fundamental tool of telemedicine, plays in these new models. The review also examines evidence of improved health care outcomes by highlighting the role of telemedicine in reducing hospitalizations. The patient is the primary focus; as a result, this review also examined patient experiences and satisfaction levels regarding telemedicine health care teams. The authors support these technological advances and their potential for information transfer. Health care providers need to continue developing these models by making use of increasing amounts of information. One of the main implementation barriers of these new models in the US and other countries is the issue of payment and reimbursement. Despite this, advancements in EC telemedicine continue.
\end{abstract}

Keywords: telemedicine, emergency care, geriatric, patient evaluation models

\section{Introduction}

Health care delivery is changing in the US and other countries as payment models change and the population ages. A primary impetus for health care change in the US is the Affordable Care Act. ${ }^{1}$ Changes in its policies and the effects of an aging population will increase demands on the health care system. ${ }^{2}$ Population management and longterm care facilities will have a much greater impact on health care systems. ${ }^{3}$ One area of particular interest for population health management is the reduction of hospital admissions and readmissions. Hospital admissions lead to increases in patient disability and higher costs. ${ }^{4}$ In addition, policy changes will lead to penalties for readmissions. ${ }^{5}$ Hospitals and health care groups are seeking methods to reduce hospitalizations through transition programs and technologies. ${ }^{6}$ New patient monitoring and communication technologies offer optimism to health care providers because they can minimize excessive use of time and costly office, emergency room (ER), and hospital visits.

One area of particular interest is telemedicine in emergency care (EC). It has the potential to dramatically improve health care issues in the home, hospital, and other health care facilities. The ability to transmit health information over distances to a
Correspondence: Paul Y Takahashi Department of Medicine, Mayo Clinic, 200 First Street SW, Rochester, MN 55905, USA

Email takahashi.paul@mayo.edu 
primary care provider or specialist can improve diagnostic accuracy and patient care without the need for a face-to-face visit. EC is often defined as medical care that requires immediate attention. This can take place during a personal visit, a phone call, or by telemedicine. The first step in EC involves telephone triage by a medical clinic or emergency response system. The present review article discusses various current EC telemedicine models, including telephone assistance. It also examines the risks and benefits of telemedicine and its impact on patients and interested parties.

\section{Telemedicine and telemonitoring in emergency care}

The American Telemedicine Association defines telemedicine as the use of medical information exchanged from one site to another by electronic communications to improve a patient's clinical health status. ${ }^{7}$ Telemedicine involves the use of technology to deliver care to a patient that is remotely located from the provider; it also provides diagnostic and clinical decision-making assistance. The basic terminology used in telemedicine services includes asynchronous home-based services (store and forward services) originating from the patient's location (originating or spoke site), and office- or hospital-based telemedicine services (distant or hub site) where the provider is located. ${ }^{7}$ Telemedicine tools include communication technologies such as telephone and video conferencing, email web services, health care portals, and telephone service providers. Hardware such as computers, smartphones, and stand-alone devices can transmit specific health information such as vital signs, radiological images, electrocardiograms (EKGs), and other patient data, in addition to verbal communications. Remote clinical information (either live or asynchronous) and additional patient data can improve patient assessment and monitoring, resulting in improved diagnosis, triage, and therapeutic interventions. Emergency medicine and acute changes in a patient's condition often require live synchronous services to provide timely feedback to patients and family members. Telemedicine services can be provided within the hospital, health care facility, or patient's home.

There are obvious circumstances in a hospital setting where telemedicine can improve access to specialty care. As health care changes, provider consolidations result in larger delivery systems. ${ }^{8}$ Many specialty practices consolidate their resources into a central hub for greater efficiency and further specialization. Health care groups use telemedicine to provide appropriate, timely, and safe care to various patient populations in different settings. In the geriatric population, common conditions for which telemedicine is feasible include stroke, acute coronary syndrome, congestive heart failure (CHF), chronic obstructive pulmonary disease (COPD), wound care, trauma, and orthopedic emergencies. ${ }^{9}$ Teleradiology has had a significant impact on EC; it has experienced early adoption and been the subject of systematic reviews on its efficacy. ${ }^{10}$ The capacity to send images to a radiologist, neurologist, or neurosurgeon has had a significant impact on senior health care in outlying medical centers.

Some of the most robust evidence for the effectiveness of telemedicine is in geriatric EC in a hospital, specifically with patients suffering from stroke or acute coronary disease. Current guidelines for appropriate treatment of thrombolysis in stroke patients include administration of thrombolytics within hours of presentation; thus, a timely diagnosis is critical for improving outcomes and avoiding the risks associated with thrombolytics. ${ }^{11}$ The need for timely evaluation is particularly relevant in remote areas that lack the expertise necessary for the diagnosis and management of stroke. Telemedicine has been successfully used to improve diagnostic accuracy, expedite transfer and reviews of imaging, and assist with management decisions regarding triage and use of thrombolytics. ${ }^{12-15}$ In a study of 2,800 Italian stroke patients, the average time for patient evaluation by telemedicine was 38 compared to 160 minutes. The authors reported a $96.5 \%$ telemedicine diagnosis accuracy rate. ${ }^{12}$ A 2012 study by Demaerschalk et al described the reliability of real-time video data from a smartphone for assessing National Institute of Health stroke scale results in 100 US stroke patients; they found a highly reliable correlation between bedside and remote neurology treatment. ${ }^{16}$ Similarly, pre-hospital transmission of EKGs in patients with acute coronary syndromes has proven to be invaluable for early diagnosis and initiation of reperfusion. Several studies have demonstrated a reduction in treatment delays among patients with myocardial infarction with elevated ST segment diagnosed by transmission of pre-hospital EKGs. ${ }^{17,18}$ It can be hypothesized that the transmission of digital images and bidirectional communications to specialist providers can assist in the diagnosis, triage, and management of other cardiac emergencies. A recent (2014) study by Kahn et al reported increased use (up to $8 \%$ ) of telemonitoring in intensive care units (ICUs) for the care of acutely-ill patients from 598 beds in 2003 to 5,799 beds in $2010 .^{19}$

Telemedicine in geriatric emergencies can also be used in trauma situations, including falls. There have been reports of successful use of PC-based workstations with cameras in rural areas that lack the expertise of trauma 
surgeons; the workstations were used to communicate with surgeons in tertiary care facilities. These providers assisted in resuscitation and stabilization of patients with major trauma, including geriatric patients. In a US pilot study of 26 patients, patients in Level-1 trauma centers treated by telemedicine had more severe injuries compared to the general population. Eighty percent of providers felt that telemedicine improved care. ${ }^{20}$ In another US study of 41 patients where telemedicine was provided to a rural area, clinicians felt that care had improved and a reduction in rural trauma care discrepancies was observed. ${ }^{21}$ Use of telemedicine in late-life mental illnesses including dementia, continues to grow. Videoconferencing with caregivers and family members can help in the management of dementia and acute mental illness. In terms of current emergency management approaches, the use of telemedicine is primarily limited to surveillance of acute events, risk management, and management of behaviors such as wandering. ${ }^{22}$ Telemedicine for mental health could also be important in long-term care. Mental health professionals could assist with depression and dementia without being physically on site and could provide guidance to caregivers.

There is a growing demand within a home to monitor patients for acute emergency changes. Wearable personal emergency response devices are commonly used by individuals at risk of falls within the home. These devices are activated when there is a fall and respond as needed. However, a meta-analysis of the effectiveness of these devices showed a lack of evidence in long-term, real-world studies. ${ }^{23}$ There is growing innovation in the field of "smart homes", where a residence is equipped with technology that, in addition to facilitating independence and quality of life, also detects adverse events in the frail elderly. These individuals might have chronic diseases such as COPD or other functional impairments, and require close monitoring but are unable to call for help in emergencies such as falls and syncope. ${ }^{24,25}$

There is varying evidence on the use of patient monitoring systems in reducing adverse outcomes in frail geriatric patients. ${ }^{26}$ In a recent pilot study of 388 US residents in seven senior communities, telemedicine enhanced rapid delivery of clinical protocols for acute care to patients with fever, shortness of breath, altered mental status, and pain. The intensive telemedicine program involved obtaining health information, physical examination results, laboratory and radiological test results, and treatment approaches used for the acute illness. The authors felt that $50 \%$ of patient visits would have required an ER visit and that telemedicine was a feasible approach for providing effective patient care. $^{27,28}$

\section{Phone management of emergency care services}

The most common device for telemedicine is the telephone. EC for the elderly often starts with a telephone call. Emergency numbers such as 911 in the US and 999 in the UK are used by all types of patients. These calls are often not simple requests for emergency transport and should be classified as telemedicine calls. The 911 "lift assist" call fits this category because it involves patient assessment from a distance without transfer to the ER. These calls are generally made by seniors who have fallen and need assistance in getting back up. A Connecticut study showed that 1,087 (4.8\%) of emergency calls to 911 between 2004 and 2009 were for lift assist. ${ }^{29}$ The telemedicine component was the transmission of vital signs and clinical assessment to the ER by emergency personnel on the scene; this information helped determine whether the patient was stable enough to remain at home after receiving assistance in getting back up.

A telephone triage call center is the entry point to $\mathrm{EC}$ for many seniors. Citizens have access to 24/7 triage call centers in several countries, including the UK, the Netherlands, Denmark, Sweden, Canada, and Australia. These call centers attempt to determine the urgency of the symptoms, and make recommendations ranging from advising the patient to call for an ambulance for immediate life-threatening emergencies or to present themselves at their local ER department for EC; they can also offer self-care advice to the patient. When the disease burden of the elderly is taken into account, calls from the elderly represent a significant percentage of all calls to these centers, and are proportionately less than calls from younger individuals. ${ }^{30}$ The largest call center in the world by published numbers is the National Health Service (NHS) 111 service (previously known as NHS Direct); it handles about 4 million calls annually. ${ }^{31}$ In 2008, NHS Direct received over 400,000 calls from individuals over 65 , with $12.3 \%$ given a recommendation for $\mathrm{EC}$; of these, a little more than half (56\%) were advised to call an ambulance and the remainder (44\%) to go to the emergency department. ${ }^{30}$ It should be noted that fewer than $1 \%$ of those calls were about falls; thus those calls, like those in the US, probably went directly to the ambulance dispatch.

A 2009 US study by North and Varkey confirmed that calls from seniors or about them were often the first contact for EC..$^{32}$ A US study of 14,646 calls made to one center found that surrogates made about $40 \%$ of calls on behalf of seniors 
over 80 years of age; $38 \%$ of those were advised to seek EC. ${ }^{33}$ The high incidence of telephone advice to the elderly to seek EC has been confirmed by hospital admission data. Almost $10 \%$ of all calls to a telephone triage center concerning subjects over 65 years of age resulted in hospitalization. ${ }^{34}$ The telephone as a telemedicine device has been around for a long time, and it continues to have a significant impact in helping the elderly seek EC and get a lift assist after a fall when EC is not needed.

\section{Benefits and uses of telemedicine in emergency care}

The potential benefits of telemedicine in EC include improved health care outcomes and patient experience, as well as cost savings. Peikes et al identified these results as part of their objectives for quality health care. ${ }^{35}$ One important primary health care outcome is hospitalizations. Many clinical trials conducted in a home setting using management of acute emergencies have examined hospitalization rate as a primary outcome. However, results of telemedicine interventions have been mixed. In a recent (2013) trial of 256 COPD patients in the UK, no differences in number of hospital admissions for COPD and time to admission were found between telemedicine and standard care patients. ${ }^{36}$ Conversely, a meta-analysis of six studies that included 604 COPD patients showed a reduction in hospitalizations for COPD patients, with an odds ratio of 0.46 (95\% confidence interval [CI]; $0.33-0.65) .{ }^{37}$ In addition, a meta-analysis of eleven telemedicine studies of CHF patients (2,710 subjects) showed a reduction in $\mathrm{CHF}$-related hospitalizations, with a relative risk of $0.79(95 \% \mathrm{CI} ; 0.67-0.94 ; P=0.008) .{ }^{38}$ As a result, the evidence for reducing hospitalizations is growing, with an emphasis on single disease-state problems. Many medical homecare models are looking at telemedicine for case management of multiple illnesses. However, a 2012 study of 205 subjects found no reductions in hospitalizations for telemonitored patients with multiple medical issues. ${ }^{26}$

A second and equally important health care outcome is improvement or stabilization of functional status. Exercise and activity can be closely monitored in patients at highest risk for EC. An exercise regimen is an important aspect of care for $\mathrm{CHF}$ patients, and has been shown to reduce hospitalizations and improve quality of life. ${ }^{39}$ The use of gait speed has become a standard measure of functional status. In a 2005 study involving 3,047 subjects, slower gait speed was found to be a predictor of adverse outcomes. ${ }^{40}$ A 2013 study showed that telemonitoring allowed for effective monitoring of gait speed in a 6 minute gait walk test. ${ }^{41}$ However, using telemedicine to encourage physical activity has shown mixed results. A 2013 study of telemedicine in 205 chronically-ill outpatients showed no improvements in functional status compared to standard care. ${ }^{42}$ However, a recent pilot study (2012) of 21 ICU patients needing follow-up physical rehabilitation showed improvements in functional status. ${ }^{43}$ There is therefore a potential for improvements in functional status such as walking speed and strength in patients who are telemonitored for EC.

An increasingly important issue that health care systems must address is the cost of implementing telemedicine versus the potential savings of reducing hospitalizations. Prior to the Affordable Care Act, there was little incentive to reduce hospitalizations; however, with potential penalties for rehospitalizations, new models to reduce hospitalizations have become viable. ${ }^{1}$ A recent study comparing six nursing homes using telemonitoring with five that were not, found reduced hospitalizations in the telemonitored homes. ${ }^{44}$ In a rudimentary 2014 economic analysis based on a reduction of 15 hospitalizations per year, nursing homes using telemedicine for evening and weekend management of acute and emergency issues had an estimated $\$ 150,000$ reduction in costs per home and a net $\$ 120,000$ gain after accounting for $\$ 30,000$ for costs of the telemedicine service. ${ }^{44}$ The most effective emergency models that resulted in cost savings used case management models for telemonitoring CHF patients. A 2010 meta-analysis reported cost savings of $35 \%-86 \%$ during study enrollment as a result of CHF telemedicine programs. ${ }^{38}$

\section{Patient, caregiver, and provider experiences}

According to the Baldridge Health Care Criteria for Performance Excellence, ${ }^{45}$ one key criterion for success is how an organization engages patients and stakeholders. Specifically, how well does a health care organization listen to patient concerns, build relationships, and use information to improve the patient experience? These organizations also identify other opportunities that can help patients achieve long-term success. Among these, patient input is important; as a result, researchers and clinical groups need to thoroughly evaluate the potential impact of telemedicine on patient outcomes. This is an understudied area, particularly in EC telemedicine.

A 2013 study by Shah et al evaluated the care of patients over 21 years of age using telemedicine in an EC setting. ${ }^{28}$ The program consisted of an in-home visit. The study included residents from six senior living communities. Satisfaction results were based on interviews conducted 
with the dispatchers, certified telemedicine assistants (CTA), telemedicine providers, participants, and caregivers at participant residences. Results showed that participants and their families were very satisfied with telemedicine based on its convenience, promptness, and completeness of evaluation. However, they said visits took longer than anticipated and that they had expected a physician to be part of the visit. From the CTA's perspective, there were some challenges in communicating with patients due to reduced resident hearing abilities and mental focus problems. These challenges were reduced by the participation of family members or caregivers. However, CTAs did not feel that they had sufficient skills or training, and this led to reports of slow response, disorganization, and discomfort.

In addition, the telemedicine team experienced technical issues such as broadband card failures, device interface failures, and software problems. These technical problems created concerns, decreased satisfaction, and increased home visit duration. Providers felt that telemonitoring improved diagnostic certainty but preferred face-to-face visits. ${ }^{28}$ One might speculate that patient and provider experiences could change with emerging technologies and improved care delivery models.

A 2003 study by Weiner et al reported a telemonitoring study to assess acute medical problems at a nursing facility. The study used a slightly younger cohort of 187 participants with a mean age of 64 . The study found that both physicians and patients were satisfied with the calls that culminated in a videoconference. These calls were mostly related to assessing changes in mental status, abnormal laboratory values, and falls. However, timely access remained an issue, since physicians often lacked immediate access to the videoconferencing equipment. ${ }^{46}$ Thus, the use of the telephone remains an important telemedicine tool for most providers. In addition, it is clear that telemedicine can add to positive patient and provider experiences when making a non-faceto-face visit.

One way to measure the quality of an experience is through a satisfaction rating system. In a study of 50 patients, patient satisfaction with telemedicine was associated with patient location (either a residence or telemonitoring site), patient educational background, and quality of verbal communication. The study indicated that 32 of 40 participants were highly satisfied with the service in their own homes; while only five of ten participants receiving telemedicine care in a nursing home gave a high rating. One hundred percent $(100 \%)$ of patients with at least a high school education gave high satisfaction ratings. Quality of verbal communication seemed to be more important than other system characteristics such as set-up time of less than 10 minutes and quality of image. ${ }^{47}$ Thus, patients and providers place a high value on the ability to have home consultations and want a meaningful discussion with the provider.

\section{Quality of life, patient satisfaction, and acceptability}

Patient satisfaction and quality of life are important aspects to consider in health care delivery models using telemedicine. The patient's personal experience is important in the value equation (patient outcomes and satisfaction over costs). A recent review article by van den Berg et al of telemedicine use in older patients found that in $38 \%$ of the 68 studies reviewed, seniors with common limitations such as cognitive, hearing, and vision difficulties were excluded from the studies. ${ }^{48}$ Thus, many of the conclusions regarding acceptability and satisfaction with telemedicine could be a reflection of a selected population and therefore biased. Regarding the effectiveness of telemedicine services in EC, a focus group of 15 senior participants evaluated this problem; they indicated that smart home technologies were helpful in obtaining emergency help. ${ }^{49}$

In general, older patients find telemedicine services acceptable and satisfying. ${ }^{27,50}$ An intensive telemedicine program including 388 patients geared toward addressing acute illnesses in individuals living in independent and assistedliving senior communities revealed both feasibility of and patient and caregiver satisfaction with the services. In this innovative program, older adults (median age 85 ) received telemedicine services for acute illnesses. These services consisted of the following: protocol-driven information gathering; collection of vital signs including pulse oximetry; visual and audio data obtained by digital camera and stethoscope; ECG; and blood and urine samples when required. This resulted in only $4 \%$ of 281 visits of patients referred to the emergency department after evaluation. However, a survey of the triage team found that, had telemedicine care not been available, care would have been recommended in either an emergency department $48.1 \%$ of the time or in an urgent care facility $27 \%$ of the time. ${ }^{27}$ Thus, the referring medical team determined that telemonitoring was useful because it provided additional information to the patients.

To include patient and caregiver perspectives in the current review, the authors performed a qualitative study of 21 participants. Results showed that telemedicine services precluded the need to travel to the ER or to an after-hours acute care center according to $91 \%$ of patients and $88 \%$ of 
caregivers. ${ }^{27}$ A high percentage (93\%) of residents agreed or strongly agreed that the service allowed comfortable interactions with the medical provider, and that they were satisfied overall with the telemedicine services. ${ }^{27}$ Overall, participants felt that the telemedicine visit was thorough. However, one drawback reported by participants was that the telemedicine visit was lengthy and some would have preferred a physician to be present in person. ${ }^{28}$

While patients and medical providers generally accept telemedicine, there are some usability considerations. A 2012 study by Chau et al examined 40 subjects using telemonitoring with a specially designed mobile phone with the capacity to contact emergency services. The participants (average age 72) reported that they were satisfied with the service; however, some reported difficulties reading the mobile phone display screen and using the small touch screen because of the fine hand movements involved. ${ }^{51}$ Technologies that are easier to use, have larger keys and text, and adjustable volumes are more likely to be accepted by older adults. ${ }^{48}$

Telemedicine has also been used as a triage tool in the emergency department. Essentially, a nurse triages a patient and has access to physician by a virtual connection. The physician can talk to and evaluate the patient, provide treatment orders, and assist with the triage process; clinical staff then expect to reduce patient wait times. A 2013 study of this technology by Traub et al in 106 patients (average age 63 years) showed that overall patient satisfaction with this service was high, with the majority of patients rating their experience as very good to excellent. They also reported high satisfaction with communications and understanding of needs. ${ }^{52}$

Telemedicine study results in older adults have been mixed, with some showing improved quality of life and others showing no effect on quality of life. In a recent review article on telemedicine in older adults, 13 studies were evaluated for quality of life: $38 \%$ showed a significant improvement in quality of life. ${ }^{48}$

\section{Summary}

The following factors have provided opportunities for the use of telemedicine in EC: an increasing number of seniors requiring health care (many with low mobility), a shift in reimbursement models to value-based purchasing, and rapidly advancing communication technologies. Care access $24 / 7$, and 365 days a year in all residential venues will become the standard of care in the next decade as organizations seek to provide timely care by the most appropriate provider. Evidence shows that telemedicine results in improved outcomes as technology improves. Patients, families, and caregivers accept and often embrace new telemedicine models because they add additional care and services to existing face-to-face models. Organizations are using telemedicine to provide primary and consultative care in locations never served before such as stroke care units in acute care hospitals and senior primary care apartments.

As with any new changes, there will always be challenges. Many of these challenges involve new care delivery models and adoption of new technologies. One such challenge involves funding for necessary clinical pilot studies to confirm the efficacy of telemedicine and to test new practice models. Public-private partnerships between providers, industry, and government resources are needed but will take time to implement. Rural practices have unique challenges and should not be excluded because of potentially lower returns on investment. Providers need to be early adopters of these sometimes disruptive, innovative, and useful technologies; they need to assess the value equation as a means of ensuring high-value care. The technology will have to demonstrate interoperability with electronic medical records, usability, dependability, affordability, and have robust data analytics and decisionsupport software. This will support the science of new health care delivery models and be considered as equal to basic and translational sciences in the crucial area of patient care.

\section{Disclosure}

Drs Takahashi, Upatising, and Hanson have received previous in-kind research support for a telemonitoring trial from GEIntel. The authors report no other conflicts of interest.

\section{References}

1. Rice T, Rosenau P, Unruh LY, Barnes AJ, Saltman RB, van Gineken E. United States of America: health system review. Health Syst Transit. 2013;15(3):1-431.

2. DeNavas-Walt $\mathrm{C}$, Proctor $\mathrm{BD}$, Lee CH. Income, Poverty, and Health Insurance Coverage in the United States: 2004. Washington, DC: US Census Bureau; 2005. Available from: http://www.census.gov/ prod/2005pubs/p60-229.pdf. Accessed June 4, 2014.

3. Leventhal T, Taliaferro JP, Wong K, Hughes C, Mun S. The patientcentered medical home and health information technology. Teleme $J E$ Health. 2012;18(2):145-149.

4. Gill TM, Allore HG, Holford TR, Guo Z. Hospitalization, restricted activity, and the development of disability among older persons. JAMA. 2004;292(17):2115-2124.

5. Kocher RP, Adashi EY. Hospital readmissions and the Affordable Care Act: paying for coordinated quality care. JAMA. 2011;306(16): 1794-1795.

6. Naylor MD, Bowles KH, McCauley KM, et al. High-value transitional care: translation of research into practice. J Eval Clin Pract. 2013;19(5): 727-733.

7. American Telemedicine Association. Home Telehealth Clinical Guidelines, 2003. Available at: http://www.americantelemed.org/ docs/default-source/standards/home-telehealth-clinical-guidelines. pdf?sfvrsn=2. Accessed May 13, 2014

8. Grauman DM, Tam MP. The urge to merge. Healthc Financ Manage. 2012;66(11):76-82, 84, 86. 
9. Brignell M, Wootton R, Gray L. The application of telemedicine to geriatric medicine. Age Ageing. 2007;36(4):369-374.

10. Hailey D, Roine R, Ohinmaa A. Systematic review of evidence for the benefits of telemedicine. J Telemed Telecare. 2002;8(1):1-30.

11. Jauch EC, Saver JL, Adams HP Jr, et al. Guidelines for the early management of patients with acute ischemic stroke: a guideline for healthcare professionals from the American Heart Association/ American Stroke Association. Stroke. 2013;44(3):870-947.

12. Angileri FF, Cardali S, Conti A, Raffa G, Tomasello F. Telemedicineassisted treatment of patients with intracerebral hemorrhage Neurosurg Focus. 2012;32(4):E6.

13. Schwamm LH, Holloway RG, Amarenco P, et al. A review of the evidence for the use of telemedicine within stroke systems of care: a scientific statement from the American Heart Association/American Stroke Association. Stroke. 2009;40(7):2616-2634.

14. Meyer BC, Demaerschalk BM. Telestroke network fundamentals. J Stroke Cerebrovasc Dis. 2012;21(7):521-529.

15. Demaerschalk BM, Raman R, Ernstrom K, Meyer BC. Efficacy of telemedicine for stroke: pooled analysis of the Stroke Team Remote Evaluation using a digital observation camera (stroke doc) and STRokE DOC Arizona telestroke trials. Telemed J E Health. 2012;18(3): 230-237.

16. Demaerschalk BM, Vegunta S, Vargas BB, Wu Q, Channer DD, Hentz JG. Reliability of real-time video smartphone for assessing National Institutes of Health Stroke Scale scores in acute stroke patients. Stroke. 2012;43(12):3271-3277.

17. Ducas RA, Philipp RK, Jassal DS, et al. Cardiac Outcomes through digital evaluation (CODE) STEMI project: prehospital digitally-assisted reperfusion strategies. Can J Cardiol. 2012;28(4):423-431.

18. Clemmensen P, Loumann-Nielsen S, Sejersten M. Telemedicine fighting acute coronary syndromes. J Electrocardiol. 2010;43(6): 615-618.

19. Kahn JM, Cicero BD, Wallace DJ, Iwashyna TJ. Adoption of ICU telemedicine in the United States. Crit Care Med. 2014;42(2): 362-368.

20. Rogers FB, Ricci M, Caputo M, et al. The use of telemedicine for realtime video consultation between trauma center and community hospita in a rural setting improves early trauma care: preliminary results. J Trauma. 2001;51(6):1037-1041.

21. Ricci MA, Caputo M, Amour J, et al. Telemedicine reduces discrepancies in rural trauma care. Telemed J E health. 2003;9(1): 3-11.

22. Westphal A, Dingjan P, Attoe R. What can low and high technologies do for late-life mental disorders? Curr Opin Psychiatry. 2010;23(6):510-515.

23. Chaudhuri S, Thompson H, Demiris G. Fall detection devices and their use with older adults: a systematic review. J Geriatr Phys Ther. Epub January 8, 2014.

24. Frisardi V, Imbimbo BP. Gerontechnology for demented patients: smart homes for smart aging. J Alzheimers Dis. 2011;23(1):143-146.

25. Hanson GJ, Takahashi PY, Pecina JL. Emerging technologies to support independent living of older adults at risk. Care Manag $J$. 2013;14(1):58-64.

26. Takahashi PY, Pecina JL, Upatising B, et al. A randomized controlled trial of telemonitoring in older adults with multiple health issues to prevent hospitalizations and emergency department visits. Arch Int Med. 2012;172(10):773-779.

27. Shah MN, Gillespie SM, Wood N, et al. High-intensity telemedicineenhanced acute care for older adults: an innovative healthcare delivery model. J Am Geriatr Soc. 2013;61(11):2000-2007.

28. Shah MN, Morris D, Jones CM, et al. A qualitative evaluation of a telemedicine-enhanced emergency care program for older adults. J Am Geriatr Soc. 2013;61(4):571-576.

29. Cone DC, Ahern J, Lee CH, Baker D, Murphy T, Bogucki S. A descriptive study of the "lift-assist" call. Prehosp Emerg Care. 2013;17(1): $51-56$.

30. Hsu WC, Bath PA, Large S, Williams S. Older people's use of NHS Direct. Age Ageing. 2011;40(3):335-340.
31. NHS Direct National Health Service Trust Annual Report \& Accounts 2012/13. HC 375. London: The Stationery Office. Available from: https://www.gov.uk/government/publications/nhs-direct-nationalhealth-service-trust-annual-report-and-accounts-2012-to-2013. Accessed May 28, 2014.

32. North F, Varkey P. A retrospective study of adult telephone triage calls in a US call centre. J Telemed Telecare. 2009;15(4):165-170.

33. North F, Muthu A, Varkey P. Differences between surrogate telephone triage calls in an adult population and self calls. $J$ Telemed Telecare. 2011;17(3):118-122.

34. North F, Varkey P. How serious are the symptoms of callers to a telephone triage call centre? J Telemed Telecare. 2010;16(7):383-388.

35. Peikes D, Zutshi A, Genevro JL, Parchman ML, Meyers DS. Early evaluations of the medical home: building on a promising start. $\mathrm{Am} \mathrm{J}$ Manage Care. 2012;18(2):105-116.

36. Pinnock H, Hanley J, McCloughan L, et al. Effectiveness of telemonitoring integrated into existing clinical services on hospital admission for exacerbation of chronic obstructive pulmonary disease: researcher blind, multicentre, randomised controlled trial. $B M J$. 2013;347:f6070.

37. McLean S, Nurmatov U, Liu JL, Pagliari C, Car J, Sheikh A. Telehealthcare for chronic obstructive pulmonary disease. Cochrane Review and meta-analysis. Br J Gen Pract. 2012;62(604):e739-749.

38. Inglis SC, Clark RA, McAlister FA, et al. Structured telephone support or telemonitoring programmes for patients with chronic heart failure. Cochrane Database Syst Rev. 2010;(8):CD007228.

39. Smart N. Exercise training for heart failure patients with and without systolic dysfunction: an evidence-based analysis of how patients benefit. Cardiol Res Pract. 2010;2011; article ID 837238.

40. Cesari M, Kritchevsky SB, Penninx BW, et al. Prognostic value of usual gait speed in well-functioning older people - results from the Health, Aging and Body Composition Study. J Am Geriatr Soc. 2005;53(10): $1675-1680$.

41. Jehn M, Prescher S, Koehler K, et al. Tele-accelerometry as a novel technique for assessing functional status in patients with heart failure: feasibility, reliability and patient safety. In J Cardiol. 2013;168(5): 4723-4728.

42. Upatising B, Hanson GJ, Kim YL, Cha SS, Yih Y, Takahashi PY. Effects of home telemonitoring on transitions between frailty states and death for older adults, a randomized controlled trial. Int J Gen Med. 2013;6:145-151

43. Jackson JC, Ely EW, Morey MC, et al. Cognitive and physical rehabilitation of intensive care unit survivors: results of the RETURN randomized controlled pilot investigation. Crit Care Med. 2012;40(4): 1088-1097.

44. Grabowski DC, O'Malley AJ. Use of telemedicine can reduce hospitalizations of nursing home residents and generate savings for medicare. Health Aff (Millwood). 2014;33(2):244-250.

45. Baldrige Performance Excellence Program. 2013. 2013-2014 Health Care Criteria for Performance Excellence. Gaithersburg, MD: US Department of Commerce, National Institute of Standards and Technology. Available from: http://www.nist.gov/baldrige. Accessed June 4, 2014.

46. Weiner M, Schadow G, Lindbergh D, et al. Clinicians' and patients' experiences and satisfaction with unscheduled, nighttime, internet-based video conferencing for assessing acute medical problems in a nursing facility. AMIA Anпu Symp Proc. 2003:709-713.

47. Chae YM, Heon Lee J, Hee Ho S, Ja Kim H, Hong Jun K, Uk Won J. Patient satisfaction with telemedicine in home health services for the elderly. Int J Med Inform. 2001;61(2-3):167-173.

48. van den Berg N, Schumann M, Kraft K, Hoffmann W. Telemedicine and telecare for older patients - a systematic review. Maturitas. 2012;73(2):94-114.

49. Demiris G, Rantz M, Aud M, et al. Older adults' attitudes towards and perceptions of "smart home" technologies: a pilot study. Med Inform Internet Med. 2004;29(2):87-94. 
50. Pecina JL, Vickers KS, Finnie DM, Hathaway JC, Hanson GJ, Takahashi PY. Telemonitoring increases patient awareness of health and prompts health-related action: initial evaluation of the TELE-ERA study. Telemed J E Health. 2011;17(6):461-466.

51. Chau JP, Lee DT, Yu DS, et al. A feasibility study to investigate the acceptability and potential effectiveness of a telecare service for older people with chronic obstructive pulmonary disease. Int J Med Inform. 2012;81(10):674-682.
52. Traub SJ, Butler R, Chang YH, Lipinski C. Emergency department physician telemedical triage. Telemed J E Health. 2013;19(11): 841-845.

\section{Publish your work in this journal}

Smart Homecare Technology and TeleHealth is an international, peer-reviewed, open access online journal publishing original research, reviews, editorials and commentaries on the application of technology to support people and patients at home and in assisted living centers to optimize healthcare and management resources. Specific topics in the journal include: Development and application of devices within the home and embedded in appliances; Healthcare provider communication and education tools; and drug ordering and adherence. The manuscript management system is completely online and includes a very quick and fair peer-review system, which is all easy to use. Visit http://www.dovepress.com/ testimonials.php to read real quotes from published authors.

Submit your manuscript here: http://www.dovepress.com/smart-homecare-technology-and-telehealth-journal 